\title{
Discovering Planar Disorder in Close-Packed Structures from X-Ray Diffraction: Beyond the Fault Model
}

\author{
D. P. Varn, ${ }^{1,2}$ G. S. Canright, ${ }^{2,3}$ and J. P. Crutchfield ${ }^{1}$ \\ ${ }^{1}$ Santa Fe Institute, 1399 Hyde Park Road, Santa Fe, NM 87501 \\ ${ }^{2}$ Department of Physics and Astronomy, University of Tennessee, Knoxville, TN 37996 \\ ${ }^{3}$ Telenor Research and Development, 1331 Fornebu, Norway
}

(Dated: February 28, 2002)

\begin{abstract}
We solve a longstanding problem - determining structural information for disordered materials from their diffraction spectra - for the case of planar disorder in close-packed structures (CPSs). Our solution offers the most complete possible statistical description of the disorder, and, from it, we find the minimum effective memory length of disordered stacking sequences. We also compare our model of disorder with the so-called fault model (FM) and demonstrate that in simple cases our approach reduces to the FM, but in cases that are more complex it provides a general and more accurate structural description than the FM. We demonstrate our technique on two previously published zinc sulphide diffraction spectra.
\end{abstract}

PACS numbers: $\quad$ 61.72.Dd, 61.10.Nz, 61.43.-j, 81.30.Hd

Santa Fe Institute Working Paper 02-03-014

Describing the structure of solids - by which we simply mean the placement of atoms in (say) a crystal - is essential to a detailed understanding of material properties. Crystallography has long used the sharp Bragg peaks in X-ray diffraction spectra to infer crystal structure. For those cases where there is diffuse scattering, however, finding - let alone describing - the structure of a solid has been more difficult [1]. Indeed, it is known that without the assumption of crystallinity, the inference problem has no unique solution [2]. Moreover, diffuse scattering implies that a solid's structure deviates from strict crystallinity. Such deviations can come in many forms - Schottky defects, substitution impurities, line dislocations, and planar disorder, to name a few. Of these, here we consider only planar disorder; that caused by one plane of atoms slipping relative to another in a layered material. This kind of disorder is known to be prevalent in a broad class of materials called polytypes.

First discovered in SiC by Baumhauer [3] in 1912, polytypes [4, 5] are solids built up from identical layers, called modular layers (MLs) [6] that differ only in their stacking orientation. A polytype is simply described by its stacking sequence - the one-dimensional list of successive orientations found as one moves along the stacking direction. We refer to the effective stochastic process induced by scanning the list as the stacking process. In the Hägg notation [5] for stacking sequences one replaces the set $\{A, B, C\}$ of allowed orientations with a binary alphabet $\mathcal{A}=\{0,1\}$ : an ML is labeled ' 1 ', if it is cyclically related to the preceding ML, or 0 , if it is not.

Polytypism is found in dozens of materials; one of the best studied is $\mathrm{ZnS}$. There are approximately 185 identified crystalline structures 顿 and many samples exhibit varying degrees of disorder. Notably, some $\mathrm{ZnS}$ crystals have unit cells extending over 100 MLs [5]. These different stacking sequences can occur under virtually identical thermodynamic growth conditions. The mystery of poly- typism then is two-fold: How can so many different structures (crystalline and noncrystalline) exist? And, what are the source and range of interlayer interactions that produce these structures? Over the last fifty years, considerable effort has been expended to understand polytypism, with over a dozen theories having been proposed; but a general explanation is still lacking [4, 5].

Attempts to describe planar disorder in CPSs have a long history. Early studies [7, \& focused on stacking errors or faults that permeated a parent crystal. Different kinds of stacking faults were postulated, such as growth faults, deformation faults, and layer displacement faults [5]. In this fault model (FM) theory, stacking faults were introduced randomly into the parent crystal and their effect on the intensity, placement, and broadening of Bragg peaks was calculated as a function of the fault frequency. These efforts met with good success for several weakly faulted specimens as such cobalt \&8 and lithium [9. However, for polytypes such as ZnS and $\mathrm{SiC}$, the random insertion of faults often did not describe the observed Bragg peaks well. More sophisticated models [5, 10, 11] were introduced which attempted to account for nonrandom fault insertion by assuming the existence of some "coordination" between faults. These more complicated models gave mixed results.

We find several drawbacks to the FM. The first is the need to assume a single parent crystalline structure into which stacking defects are introduced; this precludes the description of disorder interspersed between distinct crystal structures. In some polytypes, such as $\mathrm{ZnS}$, for example, there is considerable interest in characterizing the transformation between the hexagonally closed-packed (HCP) structure and the cubic close-packed (CCP) structure when the crystal is subjected to an external stress, such as annealing [5. In these cases, there is no single parent crystal in which to introduce faulting. A second drawback is not inherit to the FM, but in the way it is 
analyzed [5. By considering only the effects of faulting on Bragg peaks, information in the diffuse scattering is ignored. (Our second example below demonstrates how misleading this can be.) Our final difficulty is with the FM generally. Here we show that it is not possible to uniquely identify and assign faulting sequences to disordered crystals, except in those special cases to which the FM is restricted.

In this Letter, we introduce a novel method for discovering and describing disordered stacking sequences in CPSs that overcomes these difficulties. We analyze two previously published diffraction spectra for polytypic $\mathrm{ZnS}$ [5] and compare our results with those of the FM.

Our method can be broken into three parts. In the first, we use a diffraction spectrum to find average correlations between MLs as a function of the number $n$ of separating layers. If we assume that the MLs themselves are undefected, that each ML has the same scattering power, and that the spacing between MLs is independent of the local stacking arrangement, then correlation factors (CFs), $Q_{c}(n)$ and $Q_{a}(n)$ [12], can be found by Fourier analysis of the diffraction spectrum [2]. $Q_{c}(n)$ and $Q_{a}(n)$ are defined as the probability that any two MLs at separation $n$ are cyclically or anticyclically related, respectively.

In the second part of our approach, we infer the spatial patterns of MLs that reproduce these CFs by reconstructing an $\epsilon$-machine [13], which describes the minimal effective states of the stacking process. Assume we know the probability $p(\omega)$ of stacking sequences $\omega$. At each ML in a stacking sequence define the "past" $\overleftarrow{\omega}$ as those MLs already seen and the "future" $\vec{\omega}$ as those yet to be seen: $\omega=\overleftarrow{\omega} \vec{\omega}$. The effective states of the stacking process then are defined as the sets of pasts $\overleftarrow{\omega}$ that lead to statistically equivalent futures:

$$
\overleftarrow{\omega}_{i} \sim \overleftarrow{\omega}_{j} \text { if and only if } p\left(\vec{\omega} \mid \overleftarrow{\omega}_{i}\right)=p\left(\vec{\omega} \mid \overleftarrow{\omega}_{j}\right)
$$

These equivalence classes of pasts are the stacking process's causal states. Along with their transitions, they comprise the process's $\epsilon$-machine - a statistical description of the ensemble of spatial patterns that produces the stacking distribution $p(\omega)$. It has been shown that the $\epsilon$-machine is the optimal predictor of minimal size (as measured by the number of states) of a process, and, up to state-relabeling, it is the unique such description [13].

To find the causal states we must first estimate the probability $p(\omega)$ of stacking sequences $\omega$ averaged over the sample. Note that, from conservation of probability, $p(u)=p(0 u)+p(1 u)=p(u 0)+p(u 1)$, for all $u \in \mathcal{A}^{r}$, where $\mathcal{A}^{r}$ is the set of all sequences of length $r$. Additionally, the probabilities for sequences of the same length are normalized: $\sum_{\omega \in \mathcal{A}^{r+1}} p(\omega)=1$. Together these constraints provide $2^{r}$ independent relations among probabilities for the $2^{r+1}$ possible stacking sequences of length $r+1$.
The other $2^{r}$ constraints come from relating CFs to sequence probabilities via

$$
Q_{\alpha}(n)=\sum_{\omega \in \mathcal{A}_{\alpha}^{n}} p(\omega),
$$

where $\mathcal{A}_{\alpha}^{n}$ is that subset of length- $n$ sequences with a cyclic $(\alpha=c)$ or an anticyclic $(\alpha=a)$ rotation between MLs at separation $n$. We take as many of these latter relations as necessary to form a complete set of equations. At a fixed $r$, the set of equations describes the stacking sequence as generated by an $r^{\mathrm{th}}$-order Markov process. At $r=3$ one encounters the first nonlinearities due to the necessity of using CFs at $n=5$ to obtain a complete set of equations. We rewrite the probability of sequences of length $n=5$ in terms of the conditional probabilities of those at $n=4$, and it is this mapping that is nonlinear. We solve numerically for the stacking sequence probabilities $p(\omega)$ and then find the set of causal states using the equivalence relation Eq. (11). The causal-state transitions are estimated from the conditional distributions of the next ML orientation given pasts $\overleftarrow{\omega}$ associated with each causal state.

In the third and final part, we begin with the $r=1$ reconstructed $\epsilon$-machine, use it to generate a sample stacking sequence (here we used length 400,000), and from this we estimate the $\epsilon$-machine's predicted CFs and diffraction spectrum. We then compare the latter to the experimental diffraction spectrum. If there is not sufficient agreement, we increment $r$ and repeat the reconstruction and comparison. The resulting $r$ is called the stacking process's memory length, since it is the amount of history (in MLs) one must use to optimally predict the process.

ZnS can be thought to have a CPS with a basis composed of two atoms, zinc and sulphur, with the sulphurs displaced one quarter of a body diagonal (as referred to the conventional unit cell) along the stacking direction [5]. We take an ML to be this zinc sulphur pair arranged in a hexagonal net [6], giving (as with any CPS) three absolute orientations for the MLs, but only two relative orientations for neighboring layers. We correct the experimentally obtained diffraction spectrum for the atomic scattering factors, the structure factor, dispersion factors, and polarization of the incident radiation [14].

We now give the results for $\epsilon$-machine reconstruction for two experimental diffraction spectra, SK134 and SK135 from [5]. Let $l$ be a continuous variable that indexes the magnitude of the perpendicular component of the diffracted wave $k=2 \pi l / c$, where $c$ is the spacing between adjacent MLs. We select a unit interval in $l$ on which to analyze each spectra. Since many diffraction spectra suffer from experimental error [5], we show elsewhere 15 that there are relations that the CFs must obey for any CPS and that we can use them to select a relatively error-free $l$-interval. The spectra from experiment and $\epsilon$-machine reconstruction are normalized. 


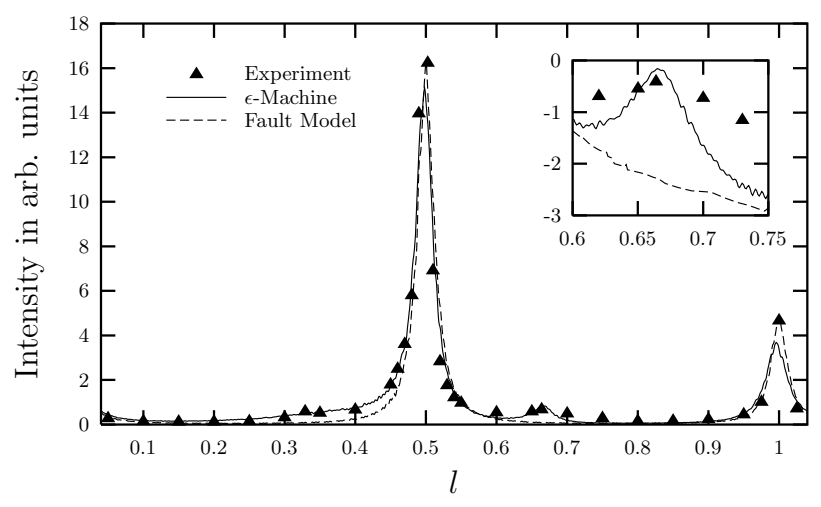

FIG. 1: Comparison of the experimental diffraction spectrum SK134 along the 10.l row (triangles) for a disordered $\mathrm{ZnS}$ single crystal [5, p. 134] with spectra estimated from the FM with $5 \%$ deformation faulting (dashed line) and $r=3$ $\epsilon$-machine (solid line). The vertical scale in the inset is logarithmic intensity.

The triangles in Fig. 1 show the experimental diffraction spectrum SK134 along the 10.l row for an HCP ZnS crystal annealed at $300 \mathrm{C}$ for one hour. Sebastian and Krishna [5] attribute the observed disorder to the introduction of $5 \%$ deformation faulting. (This is the FM predicted spectrum given as a dashed line in Fig. 1). We find that the smallest- $r$-machine that gives adequate agreement (solid line) with experiment is estimated at $r=3$; it is shown in Fig. 2 .

It is possible to give an approximate equivalent of this $\epsilon$-machine in terms of the FM, but we stress that this decomposition is not unique. We associate each closed, nonintersecting loop (called a simple cycle or SC [16]) in the $\epsilon$-machine with either a crystal structure or a fault. In this way, $\epsilon$-machines directly describe familiar structures in polytypes. For instance, the closed loop between causal states $\mathrm{C}$ and $\mathrm{H}$ in Fig. 2 implies a stacking sequence ...010101..., which is simply the Hägg notation for the HCP structure. One concludes, then, that there is no qualitative difference between what one calls faults and crystal structure. The distinction is, in fact, quantitative and one of convenience - crystal structures have relatively high probabilities, as opposed to the rarer faults. For the most general $r=3 \epsilon$-machine, it is known that there are 19 such SCs 17. Since eight independent CFs are sufficient to specify an $r=3 \epsilon$-machine, the problem of decomposing the $\epsilon$-machine into SCs is underdetermined. This conclusion holds for all $r \geq 2$. Therefore, without a fortuitous vanishing of causal states or transitions, the fault description is not unique.

For the sake of comparison with previous FM analyses, we decompose the $\epsilon$-machine in Fig. 2 into SCs with the assumption that faults corresponding to SCs of length 7 or greater are not present. We can then assign an approximate fault distribution for SK134 (second column)

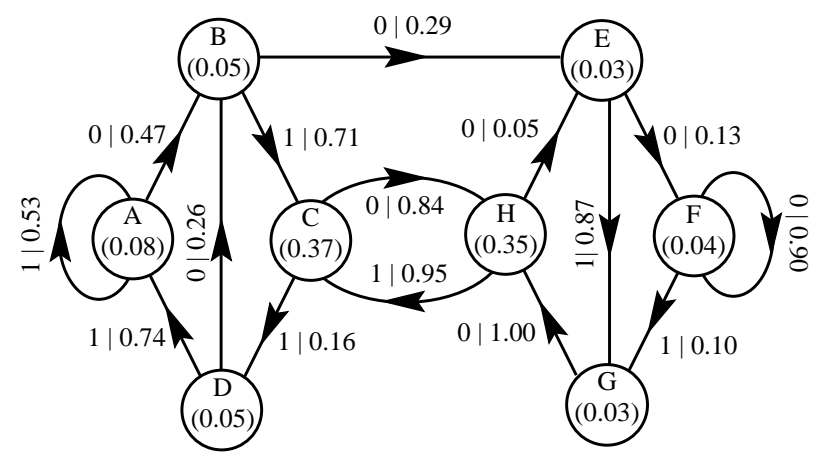

FIG. 2: The recurrent causal states $\{\mathrm{A}-\mathrm{H}\}$ of the reconstructed $\epsilon$-machine estimated from the experimental diffraction spectrum SK134 of Fig. 11 with $r=3$. Asymptotic state probabilities are given in parentheses; edge label $s \mid p$ indicates a transition on symbol $s$ with probability $p$.

as follows and compare it to that of 5 (third column):

$\begin{array}{lrr}\text { HCP } & 64 \% & 95 \% \\ \text { CCP } & 8 \% & 0 \% \\ \text { Deformation Fault } & 16 \% & 5 \% \\ \text { Growth Fault } & 6 \% & 0 \% \\ \text { Layer Displacement Fault } & 6 \% & 0 \%\end{array}$

The $\epsilon$-machine description of the crystal differs significantly from that of Sebastian and Krishna [5]. While we both find qualitatively that deformation faulting is important, we also detect CCP structures, as well as growth faults and layer displacement faults. Overall, $\epsilon-$ machine analysis finds a much more disordered crystal. This is borne out when comparing the FM and $\epsilon$-machine diffraction spectra. Fig. 11 shows that, while both agree reasonably well with experiment at the broadened peaks at $l=0.5$ and 1 , the $\epsilon$-machine is in better agreement along the shoulders of the Bragg peaks, as well as at the rise in broadband intensity at $l \approx 0.67$ (inset in Fig. 1).

Fig. 3 plots the experimental diffraction spectrum along the 10.l row (triangles) for a HCP ZnS crystal annealed at $500 \mathrm{C}$ for one hour. Sebastian and Krishna [5] calculate a twin-fault probability of $12 \%$ from the observed half-widths of the peaks. The calculated diffraction spectrum for such a faulting mechanism is shown in Fig. 3 (dashed line). Only the peak at $l=-0.33$ was used to find the faulting mechanism, and one sees that the FM reproduces it well. However, the second peak at $l=-0.67$ is poorly represented, as is the diffuse scattering between the two peaks. This demonstrates the pitfalls in simply fitting an FM to a single Bragg peak, ignoring the information contained in other peaks and in the diffuse scattering. We also note that the small rise in diffracted intensity at $l \approx-0.16$ is likewise missed by the FM. The $\epsilon$-machine spectrum (solid line) also misses this rise, but otherwise is in excellent agreement with the experiment. Fig. 目 shows the reconstructed $\epsilon$-machine 


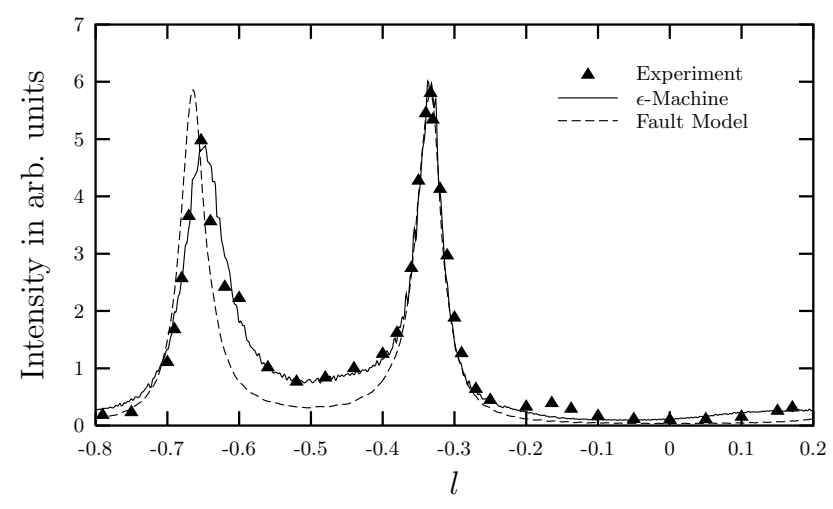

FIG. 3: Comparison of the experimental diffraction spectrum SK135 along the 10.l row (triangles) for a disordered CCP ZnS single crystal [5, p. 135] with the diffraction spectra calculated from the the FM with $12 \%$ twined faulting (dashed line) and $r=3 \epsilon$-machine (solid line).

obtained at $r=3$. The large probabilities for causal states $\mathrm{A}$ and $\mathrm{F}$ and their large self-loop transition probabilities, associated with stacking sequences ...1111... and ...0000 ..., indicate that this is a twined-CCP crystal. The missing $\mathrm{H} \rightarrow \mathrm{C}$ causal-state transition, and so the resulting absence of the ...0101 ... stacking, implies that the original HCP structure has been eliminated.

In conclusion, we presented a novel method for discovering and describing planar disorder and organization in CPSs. We demonstrated that the FM, both in conception and practice, is unable to accommodate the variety of stacking arrangements possible in nature. In contrast, $\epsilon$-machines provide a unique description of structure and can be used to quantify any amount of disorder. We argued that simply examining the effects of disordered stacking on the Bragg peaks is insufficient to properly detect the disorder present. Moreover, we quantified the memory length for disordered 1D systems; for the ZnS samples considered it was 3 MLs. Thus, the origins of polytypic ordering lie outside the physical 1D Hamilto-

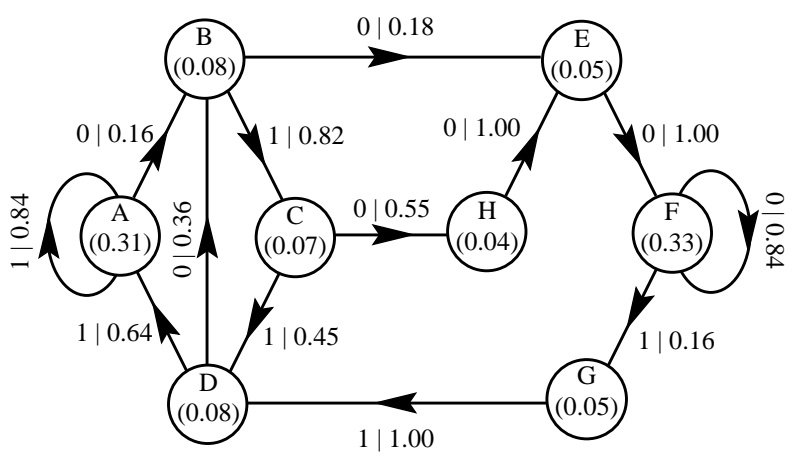

FIG. 4: Recurrent states of the reconstructed $\epsilon$-machine for the experimental diffraction data SK135 of Fig. 3 using $r=3$. nian, from which one calculates a physical interaction range of $1 \mathrm{ML}$ [18].

While we performed $\epsilon$-machine reconstruction only to $r=3$, the extension to higher $r$ is straightforward, but quite demanding computationally. Additionally, knowing the $\epsilon$-machine for a material's spatial patterns allows calculation of physically relevant quantities. We show elsewhere that given the coupling constants between MLs [18], we can determine the average stackingfault energy for a disordered crystal 15. We expect that other physical parameters will be amenable to calculation directly from $\epsilon$-machines.

The authors thank David Feldman for comments on the manuscript. This work was supported at the Santa Fe Institute under the Network Dynamics Program funded by Intel Corporation and under the Computation, Dynamics, and Inference Program via SFI's core grants from the National Science and MacArthur Foundations. Direct support was provided by NSF grants DMR-9820816 and PHY-9910217 and DARPA Agreement F30602-002-0583. DPV's visit to SFI was supported by the NSF Physics Graduate Fellows Program.

[1] T. R. Welberry, Rep. Prog. Phys. 48, 1543 (1985).

[2] A. Guinier, X-Ray Diffraction in Crystals, Imperfect Crystals and Amorphous Bodies. (W.H. Freeman and Company, San Francisco 1963).

[3] H. Baumhauer, Z. Krist. 50, 33 (1912).

[4] G. C. Trigunayat, Solid State Ionics. 48, 3 (1991).

[5] M. T. Sebastian and P. Krishna, Random, Non-Random and Periodic Faulting in Crystals. (Gordon and Breach Science Publishers, Langhorne, Pennsylvania 1994).

[6] D. P. Varn and G. S. Canright, Acta Cryst. A 57, 4 (2001).

[7] S. Hendricks and E. Teller, J. Chem. Phys. 10, 147 (1942).

[8] A. J. C. Wilson, Proc. Roy. Soc. A180, 277 (1942).

[9] R. Berliner and S. A. Werner, Phys. Rev. B 34, 3586 (1986).

[10] J. B. Gosk, Crys. Res. Tech. 35, 101 (2000).

[11] J. B. Gosk, Crys. Res. Tech. 36, 197 (2001).

[12] J. Yi and G. S. Canright, Phys. Rev. B 53, 5198 (1996).

[13] J. P. Crutchfield and K. Young, Phys. Rev. Lett. 63, 105 (1989); J. P. Crutchfield and D. P. Feldman, Phys. Rev. E 55, R1239 (1997); and C. R. Shalizi and J. P. Crutchfield, J. Stat. Phys. 104, 819 (2001).

[14] T. Hahn, A. J. C. Wilson and U. Shmueli, International Tables for Crystallography, $3^{\text {rd }}$ revised edition. (Kluwer Academic Publishers, Boston 1992).

[15] D. P. Varn, G. S. Canright and J. P. Crutchfield, in preparation. D. P. Varn. Language Extraction from $\mathrm{ZnS}$. Ph.D. Thesis, Univ. of Tenn., Knoxville (2001).

[16] G. Canright and G. Watson, J. Stat. Phys. 84, 1095 (1996).

[17] M. Teubner, Physica A 169, 407 (1990).

[18] G. E. Engel and R. J. Needs, J. Phys. Cond. Mat. 2, 367 (1990). 